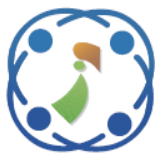

\title{
Fault Tolerant Control Based on Adaptive Fuzzy Sliding Mode Controller for Induction-Motors
}

\author{
Bachir Bendjaima $^{1 *} \quad$ Djamel Saigaa $^{1} \quad$ Djalal Eddine Khodja $^{1}$ \\ ${ }^{1}$ Department of Electrical Engineering, Faculty of Technology, \\ University Mohamed Boudiaf, M'sila, Algeria \\ * Corresponding author’s Email: zegsam5@ gmail.com
}

\begin{abstract}
In industry, the service continuity of an electrical system drives requires degrees of reliability of operation and high safety. Thus, the system requires robust elements to avoid falling into the defects. But the appearance of defects, it is inevitable. It is therefore necessary to solve this problem by developing a control technique that can ensure continuity of operation in the presence of faults. Indeed, the sliding mode adaptive control based on fuzzy logic ensures the stability of the system healthy and able to compensate certain types of defects: the defects of low severity. In fact, is with the fuzzy sliding controller with varying control gains. This one is based on supervisory fuzzy inference system using the adaptive tune in order to improve the performance of controller in presence of current sensors faults. In addition, the sensors are the most sensitive elements and the seat of frequent faults, and play a leading role in the closed loop control. An operation of a tolerant control the default intended to compensate the default offset sensor. The developed algorithm is validated by simulation and the obtained results showed the effectiveness and the robustness of the proposed approach under different scenarios.
\end{abstract}

Keywords: Adaptive control, Sliding mode control, Fuzzy logic, Fault tolerant control, Induction-motors.

\section{Introduction}

Electric drives existing in the industry are equipped with electromechanical converter (electrical machine) for energy conversion. Generally, the most of electrical drives use today the three-phase asynchronous machines [1,2].

The increased use of this type of machine has undeniably contributed in the reduction of operating costs by saving energy, and secondly to increase operational safety by the instantaneous control of various sensitive variables of the system [3 - 5].

To improve the advantage of the dependability of these machines, suitable choices of architecture strings of electrical energy conversion, their components and control strategies in normal operation and in the presence of various types of defects must be indispensable. The main objectives of the choice of components of an electromechanical conversion energy are the compactness, reliability, good dynamic performance and reducing losses [6 8].

Owing to the nonlinearities and uncertainties of asynchronous machines, the robust control algorithm is widely applied to compensate some defects, but which also exists low convergence speed problems [3, 4, 8]. In addition, research activities are focused on developing new analysis and parameters tuning methods for adaptive fuzzy controllers, which can further improve the convergence speed. The genetic algorithms are usually used to adjust the parameters of the fuzzy controller, which are improved by the adaptive mutation factors and global optimization function to guarantee the control performance $[8,9]$.

With the above mentioned motivations, this paper proposes an improved tolerant control of induction machine based on adaptive fuzzy sliding mode controller. Thus, the fuzzy sliding mode controller (FSMC) with varying control gains is presented. Specifically, a supervisory fuzzy 
inference system is used to adaptively tune the reaching control gains in order to improve the performance of the controller in presence the current sensor faults. We examine the fault-tolerant control and the response of the induction machine when the sensors fault has occurred in order to show the effectiveness and the robustness of the proposed approach under different operating conditions.

However, the presentation of this work is organized in four sections detailed below: The first section is devoted to the modelling of the motorconverter and control followed by simulation results for both modes of normal and degraded operation. The results showed deteriorating performance of our ensemble, which illustrate the importance of the problem addressed in the context of this work. Then, in the second section is presented adaptive control technique of sliding mode based on the principle of direction of the flow, and process stability Lyapunov. We analyze the simulation results and the impact of the order on the flaws.

At the end, the third section focuses on the solutions to meet the requirements regarding faults speed sensors. We develop algorithms of adaptive control by sliding mode based on fuzzy logic, using the approach of the tolerant control sensor faults. This article will be completed with a conclusion on the proposed control of asynchronous machine.

\section{IM model in healthy operation}

In the stator reference frame, the state-space model of voltage-fed induction motor is derived from the Park model. The state vector is composed of the stator current components $\left(i_{r \alpha}, i_{r \beta}\right)$, rotor flux components $\left(\Phi_{r \alpha}, \Phi_{r \beta}\right)$ and rotor rotating pulsation $\omega_{r}$, whereas a vector control is composed of the stator voltage components $\left(v_{r \alpha}, v_{r \beta}\right)$ and the external disturbance is represented by the load torque $T_{L}[10$, $11]$.

The model of the induction motor can be put in the form of state as follows:

$$
\dot{x}=f(x)+B u
$$

With:

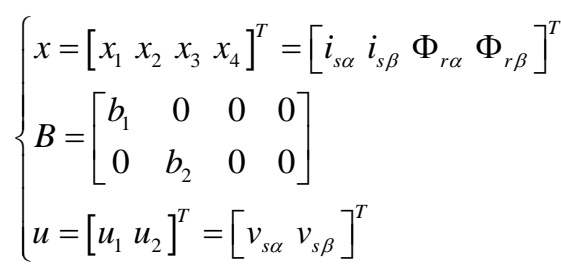

Where :

$$
\begin{aligned}
& \left\{\begin{array}{l}
\dot{x}_{1}=f_{1}(x)+b_{1} u_{1} \\
\dot{x}_{2}=f_{2}(x)+b_{2} u_{2} \\
\dot{x}_{3}=f_{3}(x) \\
\dot{x}_{4}=f_{4}(x)
\end{array}\right. \\
& \left\{\begin{array}{l}
f_{1}(x)=a_{1} x_{1}+a_{2} x_{3}+a_{3} x_{4} \omega_{r} \\
f_{2}(x)=a_{4} x_{2}+a_{5} x_{3} \omega_{r}+a_{6} x_{4} \\
f_{3}(x)=a_{7} x_{1}+a_{8} x_{3}+a_{9} x_{4} \omega_{r} \\
f_{4}(x)=a_{10} x_{2}+a_{11} x_{3} \omega_{r}+a_{12} x_{4}
\end{array}\right.
\end{aligned}
$$

And:

$\left\{\begin{array}{l}a_{1}=a_{4}=-\left(\frac{R_{s}}{\sigma L_{s}}+\frac{1-\sigma}{\sigma T_{r}}\right), a_{1}=a_{4}=\frac{1-\sigma}{M \sigma T_{r}} \\ a_{3}=\frac{M}{\sigma L_{s} L_{r}}, a_{5}=\frac{-M}{\sigma L_{s} L_{r}}, a_{7}=a_{10}=\frac{M}{T_{r}} \\ a_{8}=a_{12}=\frac{-1}{T_{r}}, a_{9}=-1, a_{11}=1, b_{1}=b_{2}=\frac{1}{\sigma L_{s}}, \omega_{r}=p \Omega_{r}\end{array}\right.$

Where $\sigma$ is the coefficient of dispersion given by: $\sigma=1-\mathrm{M}_{2} / \mathrm{Ls} \mathrm{Lr}$

$L_{s}, L_{r}, M$ are stator, rotor and mutual inductance, respectively

$R_{s}, R_{r}$ are respectively stator and rotor resistance. $T_{r}$ is the rotor time constant $\left(T_{r}=L_{r} / R_{r}\right)$.

\section{IM model in presence of faults}

The IM model in faulty operation is obtained by Adding the current sensor fault to the model (1), we obtain:

$\dot{x}=f(x)+B u+\gamma F_{s}$

With $F_{s}=\left[f_{s 1} f_{s 2}\right]^{\mathrm{T}}$ is the current sensor faults vector, $\gamma$ is the current sensor faults distribution matrix obtained by:

$\gamma=\left[\begin{array}{cc}a_{1} & 0 \\ 0 & a_{4} \\ a_{7} & 0 \\ 0 & a_{10}\end{array}\right]$

With:

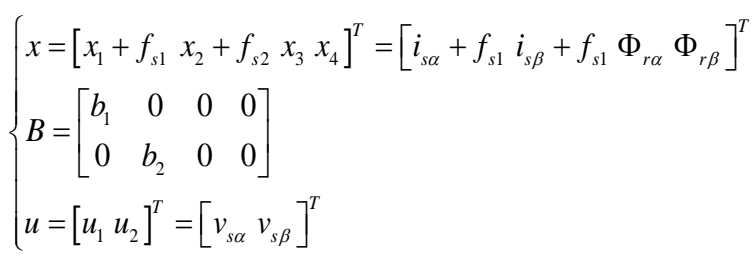


$\left\{\begin{array}{l}\dot{x}_{1}=f_{1}(x)+b_{1} u_{1}+a_{1} f_{s 1} \\ \dot{x}_{2}=f_{2}(x)+b_{2} u_{2}+a_{4} f_{s 2} \\ \dot{x}_{3}=f_{3}(x)+a_{7} f_{s 1} \\ \dot{x}_{4}=f_{4}(x)+a_{10} f_{s 2}\end{array}\right.$

\section{Assumption 1}

The resultant current sensor faults related to the IM are bounded where:

$$
\left|f_{s k}(t)\right| \leq f_{s k}^{+}, k=1,2 \quad f_{s k}^{+}>0
$$

\section{Fault tolerant based on adaptive fuzzy sliding mode controller}

\subsection{Sliding mode control design}

A Sliding Mode Controller is a variable Structure Controller (VSC). Basically, a VSC utilizes a high-speed switching control law to achieve two objectives. Firstly, it drives the nonlinear plant's state trajectory onto a specified and user-chosen surface in the state space which is called the sliding or switching surface. Secondly, it maintains the plant's state trajectory on this surface for all subsequent times [12].

Let's consider the system to be controlled described by state-space equation:

$\left\{\begin{array}{l}\dot{x}=f(x, t)+g(x, t) u \\ Y=C x\end{array}\right.$

Where $x(\mathrm{t})=\left(x_{1}, x_{2}, \ldots, x_{n}\right) \varepsilon R^{n}$ is the vector of state variables, $u(t) \varepsilon R^{n}$ is the control input $Y \in \mathfrak{R}^{p}$ is the measured output vector, $f(x, t)$ and $g(x, t)$ are both nonlinear functions.

The design of the sliding mode control needed two steps. The choice of the sliding surface, and the design of the control law.

Step1: Choice of the Sliding Surface

In [13] Slotine et al propose the general form, where it consists of a defined the scalar function for sliding surface in the phase plan. The objective is the convergence of state variable $\mathrm{x}$ at its desired value. The general formulation of the sliding surface is given by the following equation:

$$
s(x)=\sum_{i=1}^{i=n} \lambda_{i} e_{i}=e_{n}+\sum_{i=1}^{n-1} \lambda_{i} e_{i}
$$

Where $\lambda_{n}=1$, and $\lambda_{n}(i=1 \ldots n)$ present the plan coefficients.

Generally the sliding surface is given by the following linear function:

$$
S(e)=e+\lambda \dot{e}
$$

Where $\lambda$ is constant positive value, and $e=x-x_{d}$ with $x_{d}$ is the desired value.

When the commutation function is calculated, the problem of tracking needs the conception of the control law where the state vector, $e(t)$ remain close to the sliding surface. Therefore $s(x, t)=0$ for only $\mathrm{t}>=0$.

A suitable control $u$ has to be found so as to keep the error on the sliding surface, $s(e, t)=0$. To achieve this goal, a positive Lyapunov function $V$ is defined as:

$$
V(s, x, t)=\frac{1}{2} s^{2}(x, t)
$$

The sufficient condition for the stability of the system is given by:

$$
\dot{V}(s, x, t)=\dot{V}(s)=s . \dot{s}<-\eta \cdot|s|
$$

Where $\eta$ is the positive value.

Step2: control law design

The sliding mode control have two terms namely equivalent control term and switching control term:

$$
u=u_{e q}+u_{s}
$$

Where $u_{e q}$ is the equivalent part of the sliding mode control, i.e. the necessary part of the control system when $\dot{\mathrm{s}}=0, u_{s}$ describes the discontinuous control given by:

$$
u_{s}=-k \operatorname{sign}(s) \quad \text { with } k>0
$$

\subsection{Fuzzy sliding mode controller design}

In order to tackle the chattering phenomenon that is due the high frequency components, several researchers have proposed solutions allowing the reduction of these oscillations [14]:

- By increasing the commutation frequency.

- By continuous control in the band around the commutation plan.

- By auto-adjustable gain. 


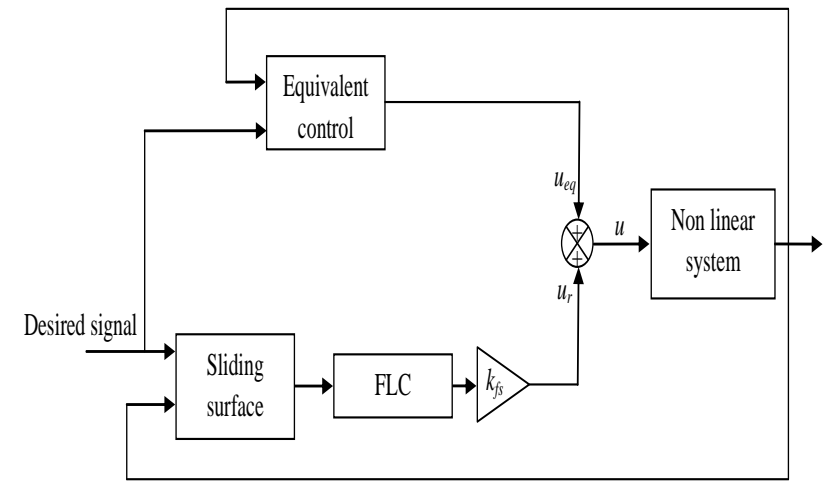

Figure.1 Block diagram of the FSMC

In the case of present work; in order to eliminate chattering phenomenon, a fuzzy logic control is used to approximate the discontinuous control. The configuration of the proposed fuzzy sliding mode control (FSMC) scheme is shown in Fig.1; it contains an equivalent control part and single input single output fuzzy logic inference system.

The equivalent control $u_{e q}$, is calculated in such a way as to have $\dot{\mathrm{s}}=0$. Then the discontinuous control is computed by:

$$
\begin{aligned}
& u_{r}=k_{f s} u_{f s} \\
& u_{f s}=F L C(s)
\end{aligned}
$$

Where $k_{f s}$ is the normalization factor of the output variable, and $u_{f s}$ is the output of the FLC, which is obtained by the normalized $s$.

The membership functions of the input sliding surface $(s)$, and the output discontinuous control $\left(u_{f s}\right)$ sets are presented by Fig. 2 .
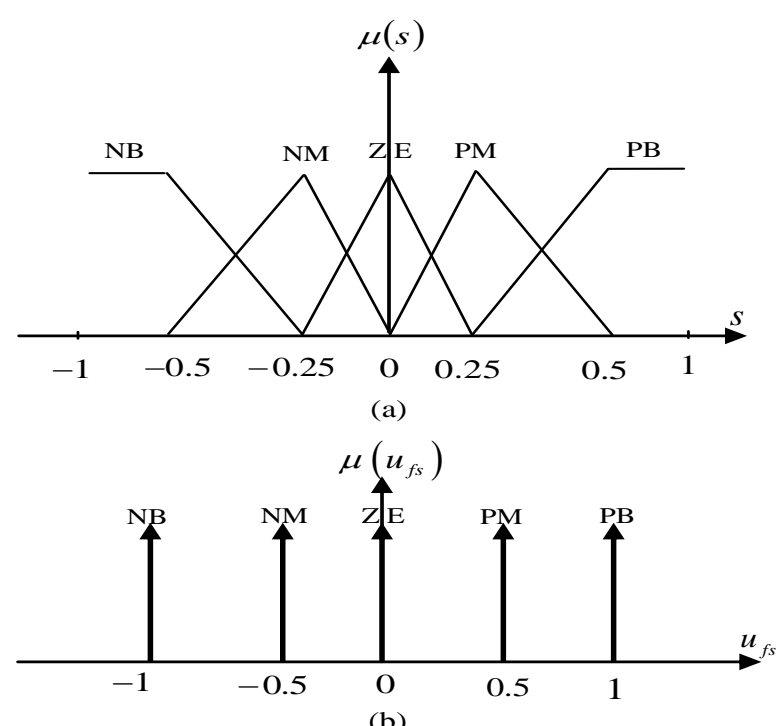

(b)

Figure.2 Membership functions of input $\mathbf{S}$ and output

$$
u_{f s}
$$

Table 1. Fuzzy rules

\begin{tabular}{|c|c|c|c|c|c|}
\hline & Rule 1 & Rule 2 & Rule 3 & Rule 4 & Rule 5 \\
\hline Surface & PB & PM & ZE & NM & NB \\
\hline$u_{f s}$ & NB & NM & ZE & PM & PB \\
\hline
\end{tabular}

In order to attenuate the chattering effect and handle the uncertainty of the six rotors helicopter, a fuzzy controller has been used with single input and single output for each subsystem. Then, the input of the controller is the sliding surface and the output is the discontinuous control $u_{f s}$. All the membership functions of the fuzzy input variable are chosen to be triangular and trapezoidal for all upper and lower membership functions. The used labels of the fuzzy variable (surface) are: \{negative medium (NM), negative big (NB), zero ( $\mathrm{ZE})$, positive medium (NM), positive big (PB)\}.

The corrective control is decomposed into five levels represented by a set of linguistic variables: negative big (NB), negative medium (NM), zero $(\mathrm{ZE})$, positive medium (PM) and positive big (PB). Table. 1 presents the rules base which contains five rules:

The membership functions of the input (sliding surface) and output $\left(u_{f s}\right)$ has been normalized in the interval $[-1,1]$, therefore: I $u_{f s}$ $\mathrm{k}<=1$. $u_{f s}$ given in equation (19) satisfies the following condition

$$
s u_{f s}=-K^{+}|s|
$$

Where $\mathrm{K}^{+}>0$ is positive constant determined by a fuzzy inference system.

In Fig. 2 the control law is computed by:

$u=u_{e q}+u_{r}=u_{e q}+k_{f s} u_{f s}$

\subsection{Fuzzy sliding mode controller design}

\subsubsection{Speed control}

To control the speed of the induction motor, we take the surface proposed by J.J.Slotine [13], the speed control manifold equations can be obtained as:

$$
S_{1}=K_{1} \cdot\left(\Omega_{r}-\Omega_{r e f}\right)+\left(\dot{\Omega}_{r}-\dot{\Omega}_{r e f}\right)
$$

Whose corresponding time derivative is:

$$
\dot{S}_{1}=K_{1} \cdot\left(\dot{\Omega}_{r}-\dot{\Omega}_{r e f}\right)+\left(\ddot{\Omega}_{r}-\ddot{\Omega}_{r e f}\right)
$$

\subsubsection{Flux control}

Similarly the sliding surface of flow is introduced as follows: 


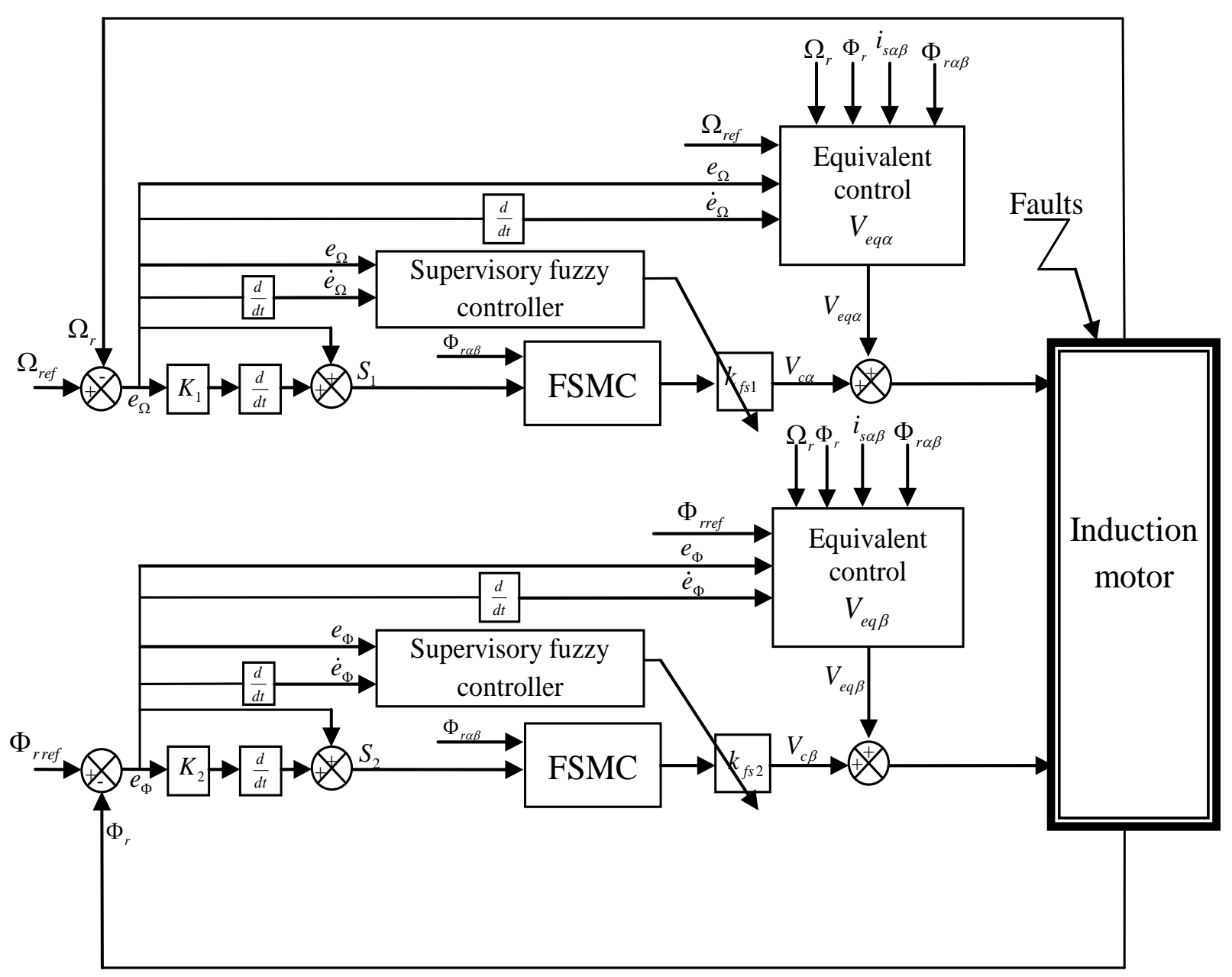

Figure.3 Block diagram of the fault tolerant control based on adaptive fuzzy sliding mode control

$$
S_{2}=K_{2} \cdot\left(\Phi_{r}-\Phi_{r r e f}\right)+\left(\dot{\Phi}_{r}-\dot{\Phi}_{r e f}\right)
$$

Whose corresponding time derivative is:

$$
\dot{S}_{2}=K_{2} \cdot\left(\dot{\Phi}_{r}-\dot{\Phi}_{r e f}\right)+\left(\ddot{\Phi}_{r}-\ddot{\Phi}_{r e f}\right)
$$

After simplification calculates derivatives of the sliding surfaces are given in matrix form as follows:

$$
\left[\begin{array}{c}
\dot{S}_{1} \\
\dot{S}_{2}
\end{array}\right]=\left[\begin{array}{c}
F_{1} \\
F_{2}
\end{array}\right]+\left[\begin{array}{cc}
-b_{2} \Phi_{r \beta} & b_{1} \Phi_{r \alpha} \\
b_{1} M \Phi_{r \alpha} & b_{2} M \Phi_{r \beta}
\end{array}\right] \cdot\left[\begin{array}{c}
V_{s \alpha} \\
V_{s \beta}
\end{array}\right]
$$

With:

$$
\left\{\begin{array}{l}
F_{1}=\left(K_{1}-a_{5}-a_{1}\right) a_{7} h_{2}-K_{1} a_{8}-\Omega_{r} a_{7}\left(h_{1}+a_{3} \Phi_{r}\right)- \\
K_{1} \dot{\Omega}_{r e f}-\ddot{\Omega}_{r e f} \\
F_{2}=2 a_{5}\left(\frac{2+M \sigma}{T_{r}}-K_{2}\right) \Phi_{r}+2 a_{4}\left(\frac{M}{T_{r}} h_{3}+\right. \\
\left.\left(K_{2}-\frac{3}{T_{r}}-a_{1}\right) h_{1}+p \Omega_{r} h_{2}\right)-K_{2} \dot{\Phi}_{r e f}-\frac{T_{r}}{2} \ddot{\Phi}_{r e f}
\end{array}\right.
$$

Where :

$$
\left\{\begin{array}{l}
h_{1}=i_{s \alpha} \Phi_{r \alpha}+i_{s \beta} \Phi_{r \beta} \\
h_{2}=i_{s \beta} \Phi_{r \alpha}-i_{s \alpha} \Phi_{r \beta} \\
h_{3}=i_{s \alpha}^{2}+i_{s \beta}^{2}
\end{array}\right.
$$

In the condensed form (26) is rewritten by:

$$
\dot{S}=F+D \cdot V_{s \alpha \beta}
$$

Where: $F=\left[\begin{array}{l}F_{1} \\ F_{2}\end{array}\right]$ and $D=\left[\begin{array}{cc}-b_{2} \Phi_{r \beta} & b_{1} \Phi_{r \alpha} \\ b_{1} M \Phi_{r \alpha} & b_{2} M \Phi_{r \beta}\end{array}\right]$

And check the condition of Lyapunov stability $(S \dot{S}<0)$, must have:

$$
\dot{S}=-k_{f s} F L C(S)
$$

Equating (29) and (30) we have:

$$
V_{s \alpha \beta}=-D^{-1} k_{f s} F L C(S)-D^{-1} \cdot F
$$


Table 2. Rule matrix of supervisory fuzzy control

\begin{tabular}{|c|c|c|c|c|c|c|c|}
\hline \multirow{2}{*}{$\begin{array}{c}k_{\mathrm{fs} 1} \text { and } \\
k_{\mathrm{fs} 2} \\
\dot{\mathrm{e}}_{\Omega}, \dot{\mathrm{e}}_{\Phi}\end{array}$} & \multicolumn{7}{|c|}{$e_{\Omega}, e_{\Phi}$} \\
\hline & NB & NM & NS & $\mathbf{Z}$ & PS & PM & PB \\
\hline NB & 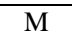 & $S$ & VS & VVS & VS & $\mathrm{S}$ & $M$ \\
\hline NM & B & $M$ & $\mathrm{~S}$ & VS & $\mathrm{S}$ & $M$ & B \\
\hline NS & VB & B & $\mathrm{M}$ & $S$ & $\mathrm{M}$ & B & VB \\
\hline $\mathbf{Z}$ & VVB & VB & $\mathrm{B}$ & $M$ & $\mathrm{~B}$ & VB & VVB \\
\hline PS & VB & B & $\mathrm{M}$ & $\mathrm{S}$ & $\mathrm{M}$ & B & VB \\
\hline PM & B & M & $S$ & VS & $\mathrm{S}$ & $\mathrm{M}$ & B \\
\hline PB & M & $\mathrm{S}$ & VS & VVS & VS & $\mathrm{S}$ & $M$ \\
\hline
\end{tabular}

The law equivalent control is given by:

$$
\left[\begin{array}{l}
V_{e q \alpha} \\
V_{e q \beta}
\end{array}\right]=-D^{-1}\left[\begin{array}{l}
F_{1} \\
F_{2}
\end{array}\right]
$$

The law of attractive control is given by:

$$
\left[\begin{array}{c}
V_{c \alpha} \\
V_{c \beta}
\end{array}\right]=-D^{-1}\left[\begin{array}{cc}
k_{f s 1} & 0 \\
0 & k_{f s 2}
\end{array}\right]\left[\begin{array}{l}
F L C\left(S_{1}\right) \\
F L C\left(S_{2}\right)
\end{array}\right]
$$

Where :

$K_{1}$ and $K_{2}$ are positive gains.

$$
k_{f s 1}>\left|F_{1}\right|, k_{f s 2}>\left|F_{2}\right|
$$

Selecting the gains $K_{1}$ and $K_{2}$ is such that the desired value is imposed at the output of each regulator [10]. The global control ensuring both $\left(S_{1}=0, S_{2}=0, S_{1} \dot{S}_{1}<0\right.$ and $\left.S_{2} \dot{S}_{2}<0\right)$ is given by:

$$
V_{s \alpha \beta}=\left[\begin{array}{c}
V_{s \alpha} \\
V_{s \beta}
\end{array}\right]=\left[\begin{array}{c}
V_{e q \alpha} \\
V_{e q \beta}
\end{array}\right]+\left[\begin{array}{c}
V_{c \alpha} \\
V_{c \beta}
\end{array}\right]
$$

\subsubsection{Adaptive fuzzy sliding mode controller}

In this section, the fuzzy sliding mode controller (FSMC) with varying control gains is presented. The general structure of the proposed controller is given in Fig.3. Specifically, a supervisory fuzzy inference system is used to adaptively tune the reaching control gains $k_{\text {fsi }}$ for $i=1,2$ in order to improve the performance of the controller in presence the current sensor faults .

The supervisory fuzzy system of the proposed tuning method contains operator knowledge in the form of IF-THEN rules to decide the control gains $k_{\mathrm{fsi}}$ for $i=1,2$ according to the current operating conditions of the controlled system. Here, the control rules of the supervisory fuzzy system of the gain $k_{\mathrm{fs} 1}$ is developed with the error $e_{\Omega}=\Omega_{r}-\Omega_{\text {rref }}$ and derivative of error $\dot{\mathrm{e}}_{\Omega}$ as a premise, and the control rules of the supervisory fuzzy system of the gain $k_{\mathrm{fs} 2}$ is developed with the error $e_{\Phi}=\Phi_{r}-\Phi_{r e f}$ and derivative of error $\dot{\mathrm{e}}_{\Phi}$ as a premise. Figs. 4-7 shows the fuzzy control surface of $k_{\mathrm{fsi}}$ for $i=1,2$. This surface has been used to adaptively tune the gains $k_{\mathrm{fsi}}$ on line. The physical domain of the inputs $\left(e_{\Omega}, \dot{e}_{\Omega}\right)$ and $\left(e_{\Phi}, \dot{e}_{\Phi}\right)$ is in the range $\{-0.01$, $0.01\}$ and that of the output $k_{\mathrm{fsi}}$ for $i=1,2$ is in the range $\{30000,50000\}$ and $\{10000,20000\}$, respectively, selected based on trial and error approach. The fuzzy variables are defined for the rule base as:

$\left(e_{\Omega}, \dot{\mathrm{e}}_{\Omega}\right)$ and $\left(e_{\Phi}, \dot{\mathrm{e}}_{\Phi}\right)=\{\mathrm{NB}($ Negative Big $)$, NM(Negative Medium), NS(Negative Small), Z(Zero), PS(Positive Small), PN(Positive Medium), PB(Positive Big)\};

$k_{\mathrm{fs} 1}$ and $k_{\mathrm{fs} 2}=$ \{VVS (Very Very Small), VS (Very Small), S (Small), M (Medium), B (Big), VB (Very Big) and VVB (Very Very Big). The linguistic fuzzy rules of the supervisory fuzzy system are given in Table 2 .

\section{Simulation results and discussion}

In this section is presented the simulation of the fuzzy sliding mode control where, Fig.8 illustrate the simulation results developed for the performance of the fuzzy sliding mode control where a current sensor faults $i_{s \alpha}$ and $i_{s \beta}$ are added in the induction motor at instants $2 \mathrm{~s}$, where the evolution of the current sensor faults are given in Fig.8.b and Fig.8.c. The different physical parameters of the induction motor are given in Table. 3 presented in the appendix.

From these results, we can see that the fuzzy sliding mode control present a good tracking of the desired trajectories on the speed and flux, but is insufficient in case of faults. Increased strength reduces the error on the speed and flux but does not negate the effect of the current sensor faults. Thus, in order to improve again the control of this assembly, in the next section is presented the Simulation of the fault tolerant control based on adaptive fuzzy sliding mode control. In fact, Fig.9 illustrate the simulation results developed for the performance of fault tolerant control based on adaptive fuzzy sliding mode control where a current sensor faults in $i_{s \alpha}$ and $i_{s \beta}$ are added in the induction motor at instants $2 \mathrm{~s}$, where the evolution of the current sensor faults are given in Fig.9.b and Fig.9.c. 


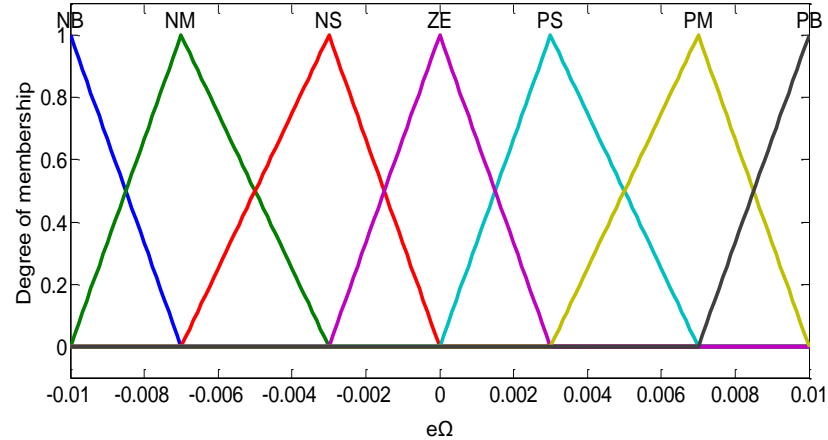

(a)

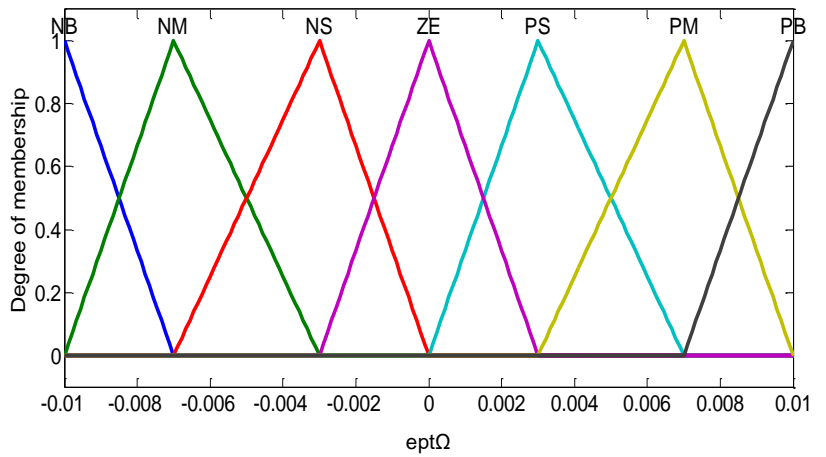

(b)

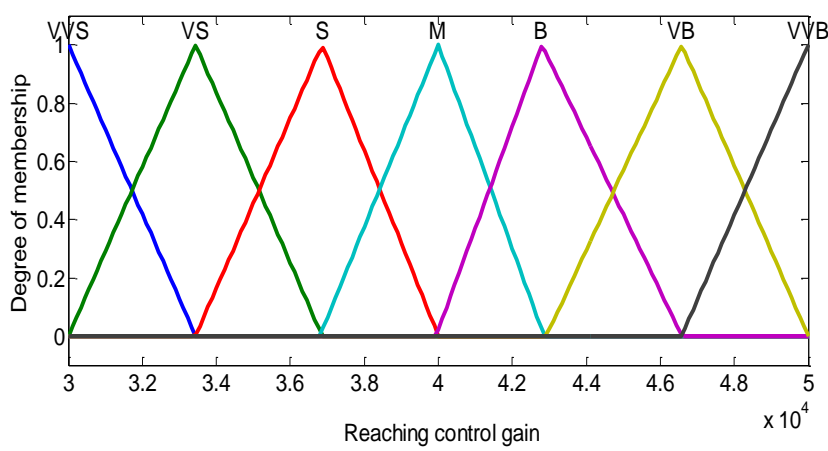

(c)

Figure.4 Membership functions of $k_{f s 1}$

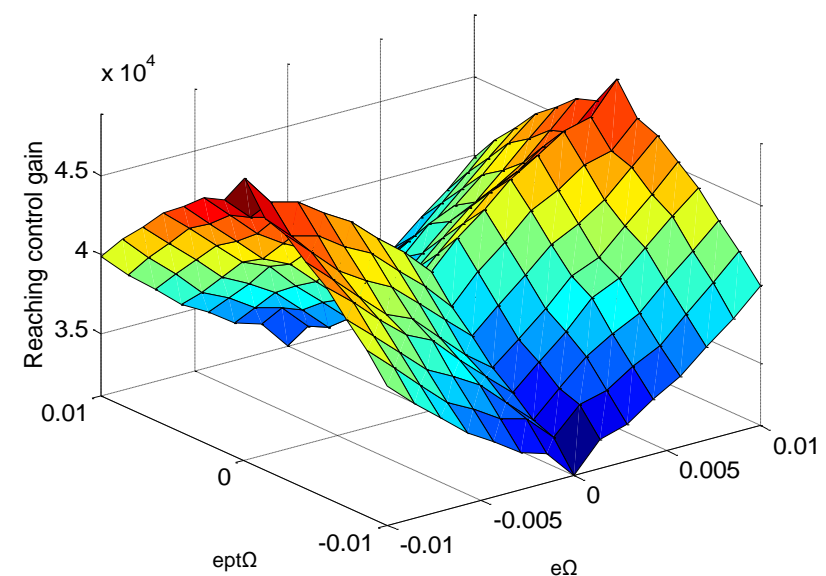

Figure.5 Fuzzy control surfaces of $k_{f s 1}$

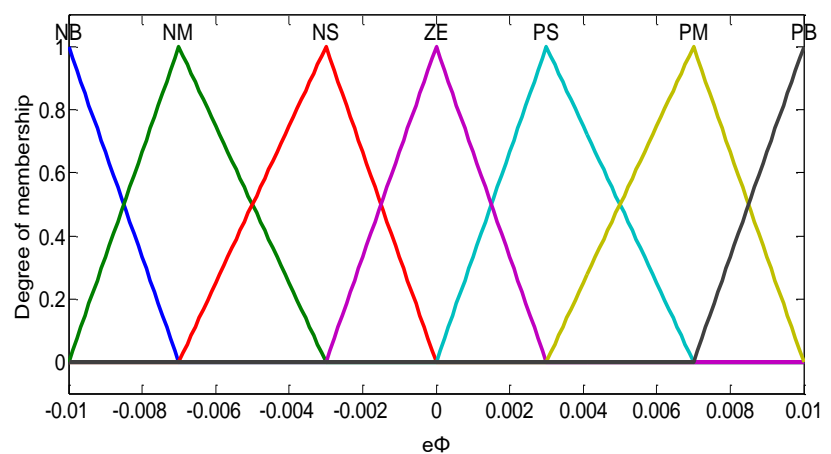

(a)

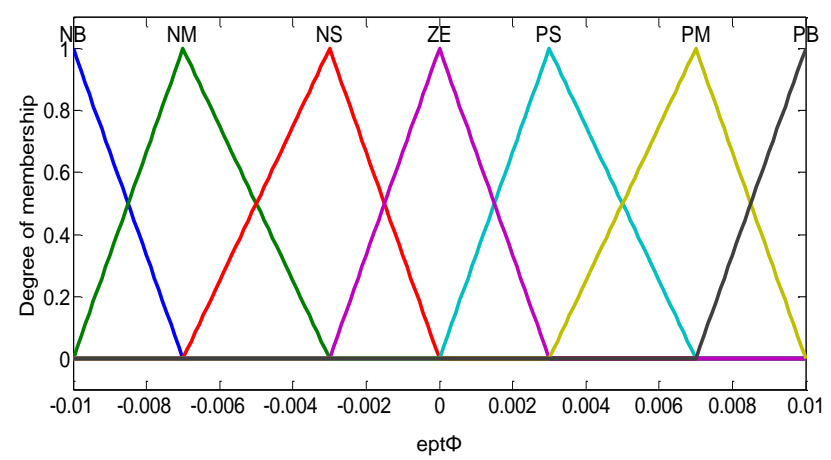

(b)

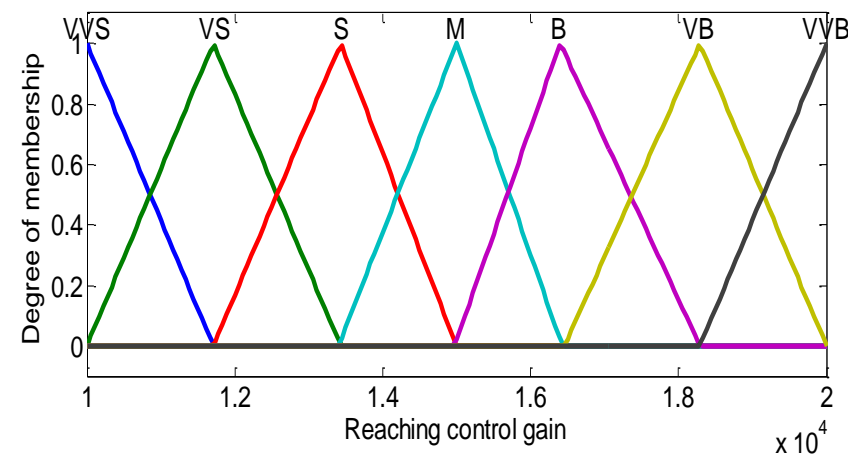

(c)

Figure.6 Membership functions of $k_{f s 2}$

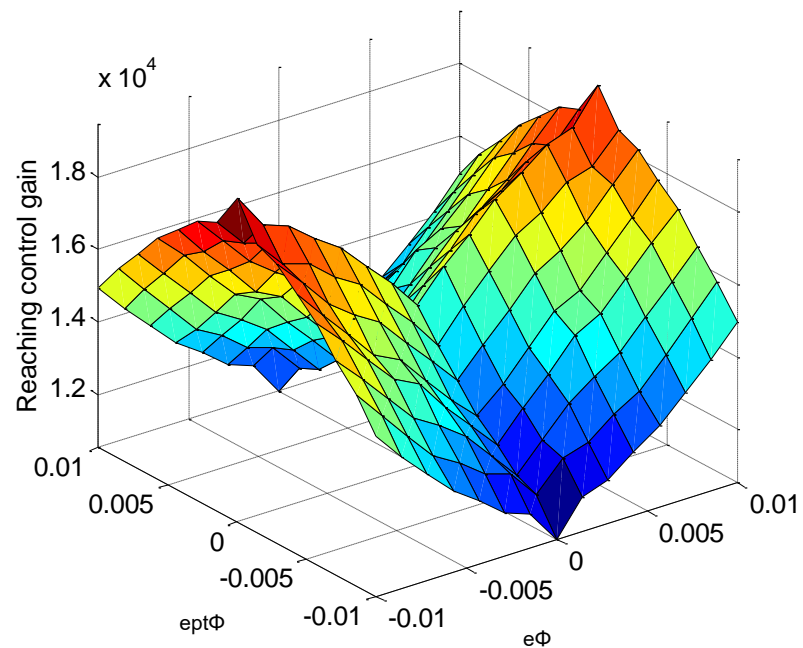

Figure.7 Fuzzy control surfaces of $k_{f s 2}$ 


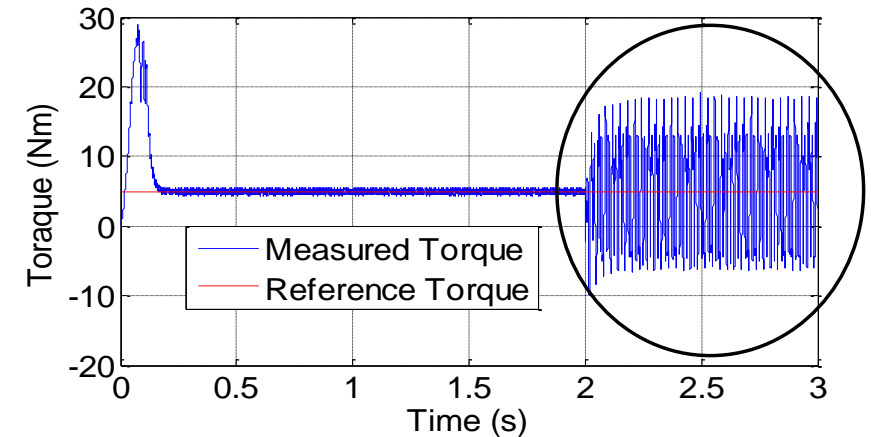

(a)

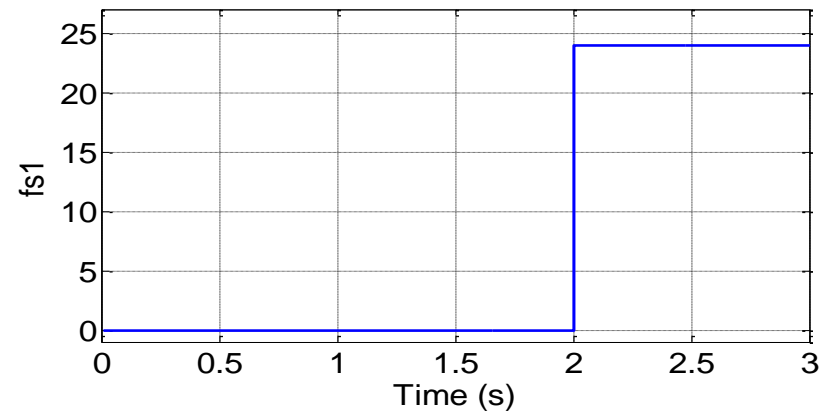

(b)

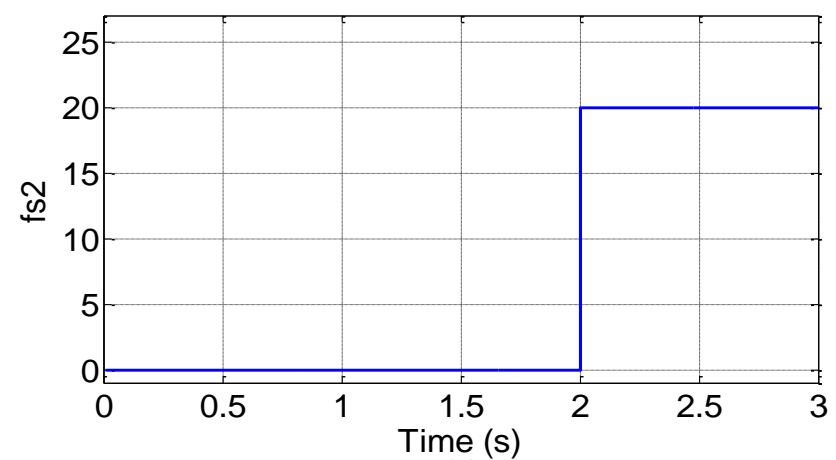

(c)

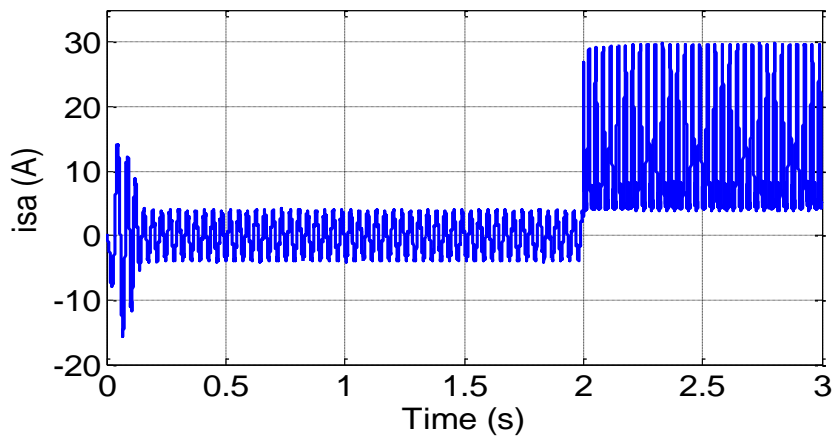

(d)

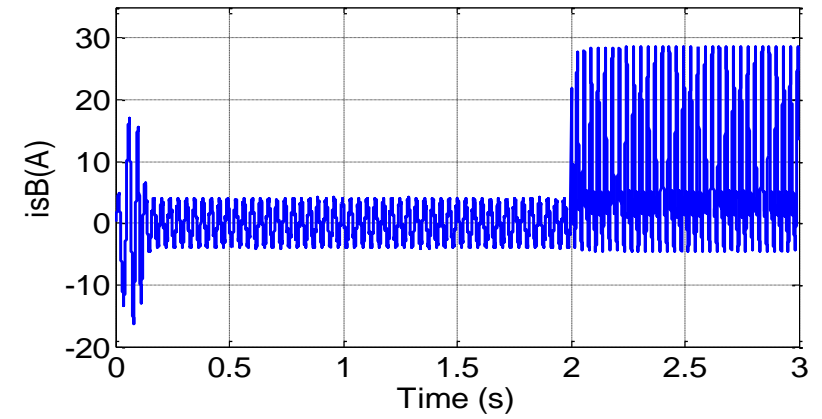

(e)

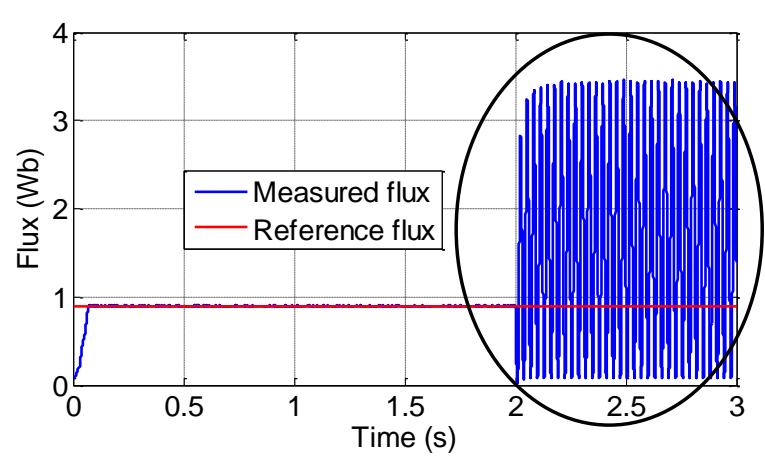

(f)

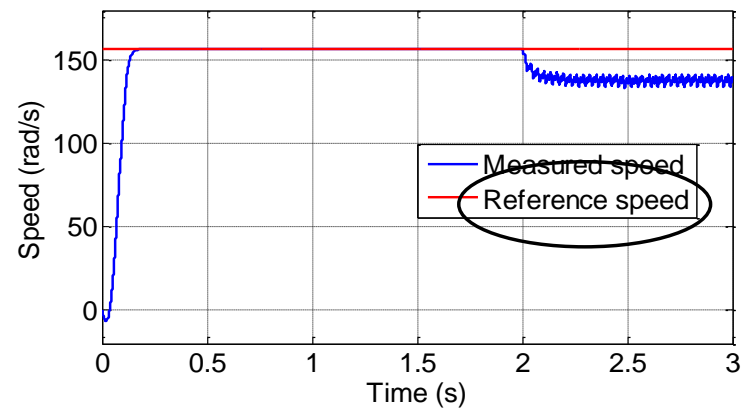

(g)

Figure.8 Simulation results of the fuzzy sliding mode control

The results show the evolution of variables respectively the electromagnetic torque, the stator current phase, the mechanical speed and flux. In figure. 8 , the speed and the flux trajectories converge to their desired references and the load disturbance is rejected. Figures.9, show the responses of fuzzy sliding mode control for the induction motor with the fault current sensors in phase A. note that the speed tracks the reference value adequately in case of healthy condition. We observe the absence of severe oscillations in the electromagnetic torque which were registered with sliding mode control [15]. Indeed, the fault tolerant control compensates the effects of these faults introduced at time $\mathrm{t}=2 \mathrm{sec}$.

According to Fig.9 we can see that the proposed fault tolerant control based on adaptive fuzzy sliding mode controller is able to make the induction motor follow the reference trajectory in torque, flux and speed, thus demonstrating the robustness of the proposed controller. Moreover, the effects of the current sensor faults are not visible on the tracking performance. This, once again, demonstrates the robustness of the proposed controller in faulty operation. 


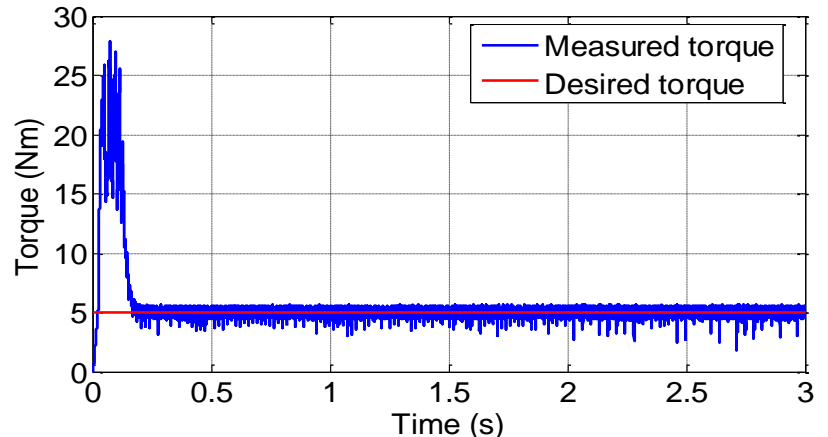

(a)

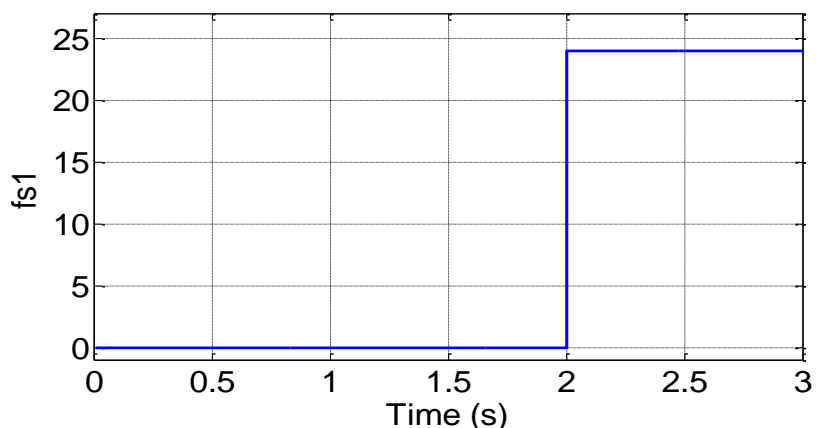

(b)

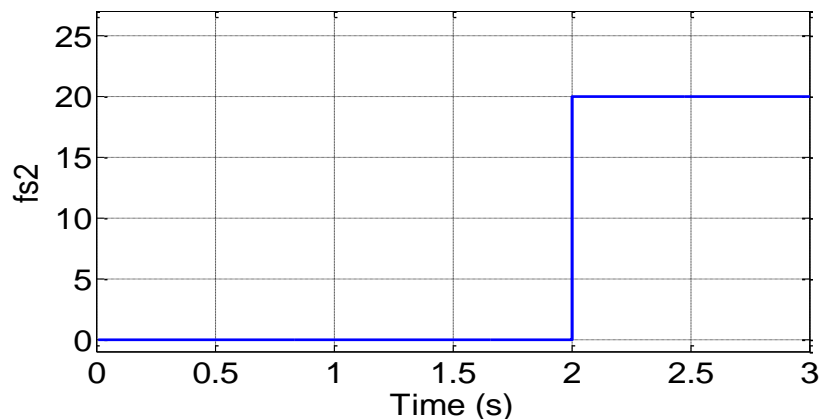

(c)

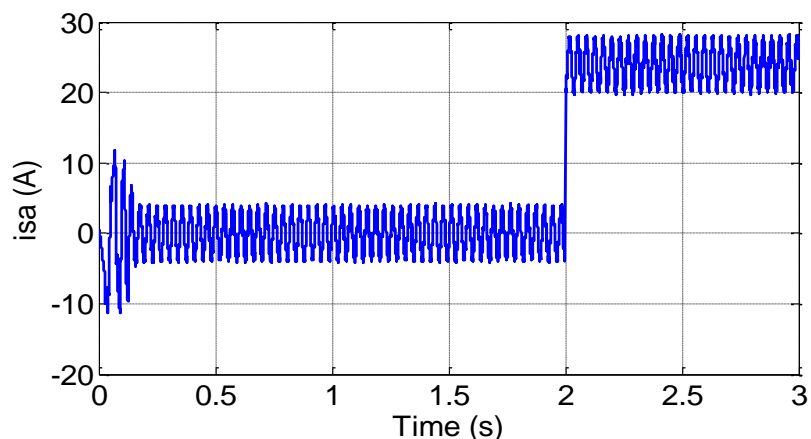

(d)

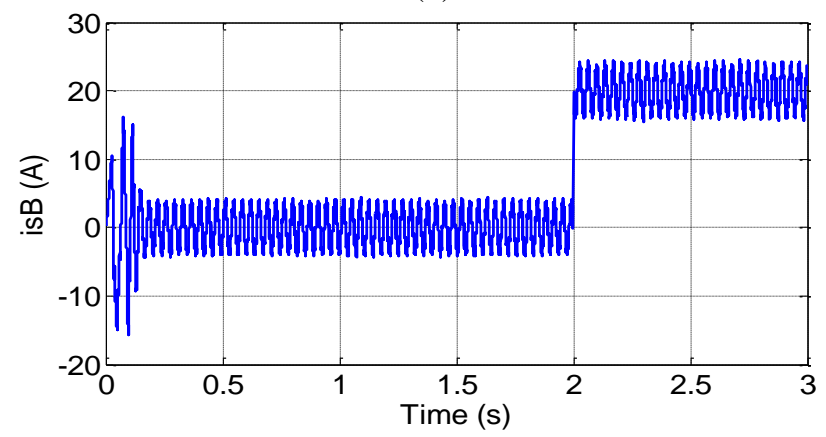

(e)

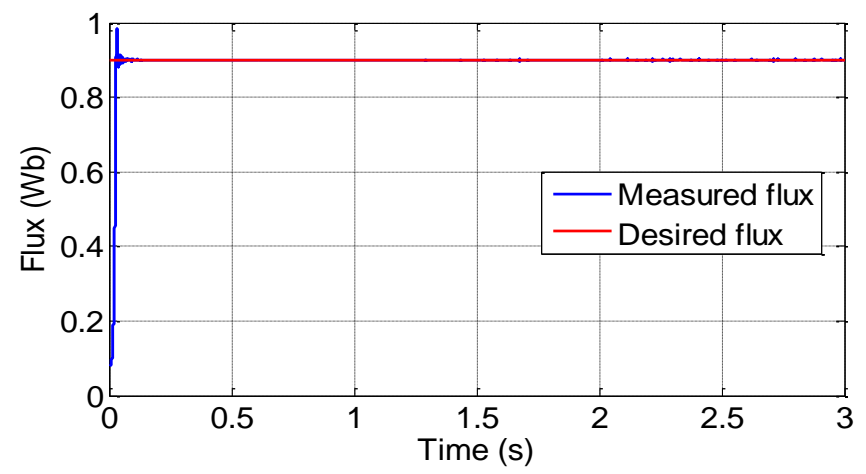

(f)

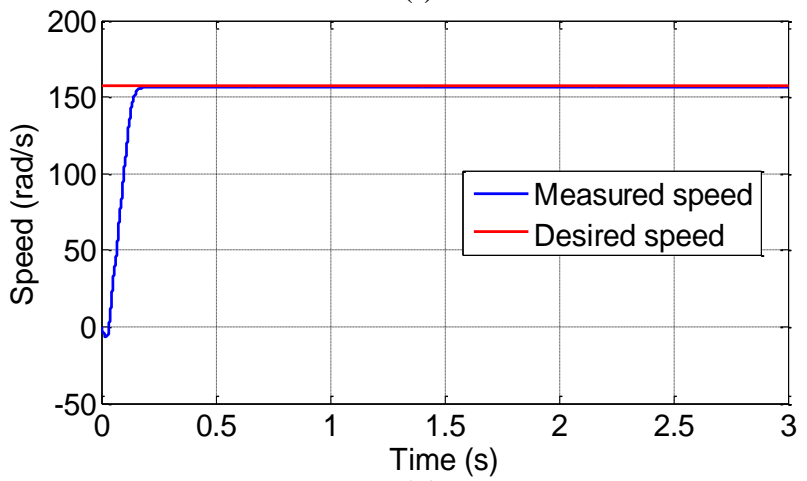

(g)

Figure.9 Simulation results of the fault tolerant control based on adaptive fuzzy sliding mode control

\section{Conclusion}

This paper has proposed a fault-tolerant control of induction motor based on adaptive fuzzy sliding mode control strategy suitable for dealing with current sensor faults.

The objective was to see the contribution of fault-tolerant control in the offsetting the effects of faults on the performance of the IM.

In order to improve the performance of the fuzzy sliding mode control in presence of the faults in the system, the hitting control gain was tuned on-line according to error states of the system by a supervisory fuzzy controller, simulation results validate that the presented adaptive fuzzy sliding mode control has a satisfactory tracking performance during a malfunctions.

In fact, the proposed fault tolerant control based on adaptive fuzzy sliding mode controller is able to make the induction motor follow the reference trajectory flux and speed, thus demonstrating the robustness of the proposed controller.

The future trends will concern modelling and fault tolerant control when defects are multiples. In this case, it must be to elaborate the motor faults model with structured parameters and synthetised by the robust techniques. 


\section{Appendix}

Table. 3: Rated data of the simulated induction motor

$1.08 \mathrm{KW}, 5 \mathrm{Nm}, p=2, R_{s}=10 \Omega, R_{r}=6.3 \Omega$, $L_{s}=0.4641 \mathrm{H}, L_{r}=0.4612 \mathrm{H}, M=0.4212 \mathrm{H}, J=0.02$ $\mathrm{Kgm}^{2}, f=0.01 \mathrm{Nmsec}$

\section{References}

[1] A. Bouscayrol, B. Davat, B. de Fornel, B. François, J. P. Hautier, F. Meibody-Tabar, and M. Pietrzak-David, "Multi-machine multiconverter systems: applications to electromechanical drives", EPJ Applied Physics, Vol.10, No.2, pp.131-147, 2000.

[2] D.J. Khodja and A. Kheldoun, "Three-Phase Induction Machine Modelling Taking The Stator Faults Into Account", World Congress on Science, Engineering Technology, WCSET2009, Rome, Italy, pp 124-127, 2009.

[3] A. Ebrahim and G. Murphy, "Adaptive backstepping control of an induction motor under time-varying load torque and rotor resistance uncertainty", In: Proc. of the IEEE SSST'06, Cookeville, USA, pp. 512-518, 2006.

[4] J. Jiang and Y.Zhang, "Graceful performance dégradation in active fault tolerant control systems", In: Proc. of the 15th IFAC World Congress b'02, Barcelona, Spain, 2002.

[5] W. T. Thomson, "A review of on-line condition monitoring techniques for three-phase Squirrel cage induction motors-past, present and future", In: Proc. of the 2nd IEEE International Symposium on Diagnostics for Electrical Machines, Power Electronics and Drives SDEMPED '99, Gijon, Spain, pp. 3-17, 1999.

[6] F. Richardeau, P.Baudesson, and T.A.Meynard, "Failures-tolerance and remedial strategies of a PWM multicell inverter", IEEE Transactions on Power Electronics, Vol.17, No.6, pp. 905912, 2002.

[7] V. Smet, F. Forest, J. Huselstein, F. Richardeau, Z. Khatir, S. Lefebvre, and M. Berkani, "Ageing and Failure Modes of IGBT Modules in High-Temperature Power Cycling", IEEE Trans. Ind. Electron., Vol. 58, No. 10, pp. 4931-4941, 2011.

[8] S. Drid, M. Tadjine, and M.S. Nait-Said, "Robust backstepping vector control for the doubly fed induction motor", IET Control Theory \& Applications, Vol. 1, No.4, pp. 861868, 2007.

[9] Y. Zhang and J. Jiang, "Fault tolerant control systems design with consideration of performance degradation", In: Proc. of the American Control Conference, Arlington, VA, USA, pp 2694-2699, 2001.

[10] P.C. Krause, O. Wasynczuk, and S.D. Sudhoff, Analysis of Electric Machinery and Drive Systems, Edition.2,Wiley-Interscience, New York, N.Y.2002.

[11] W. Leonhard, Control of Electrical Drives, Edition.2, Springer-Verlag, Berlin Heidelberg, B.H. 1996.

[12] J. Liu and X. Wang, Advanced sliding mode control for mechanical systems, Edition.1, Springer-Verlag, Berlin Heidelberg, B.H.2011.

[13] J.J. Slotine and W. Li, Applied nonlinear control, Edition.1, Prentice Hall, Englewood Cliff New Jersey, E.C.N.J.1991.

[14] O. Khalal, A. Mellit, M. Rahim, H. Salhi, and A. Guessoum, "Robust control of manipulator robot by using the variable structure control with sliding mode", In: Proc. of the IEEE Mediterranean conference on Control \& Automation, Athens, Greece, pp.1-6, 2007.

[15] M. Bedboudi, H. Kherfane, D.J. Khodja, and S. Moreau, "Sliding mode based fault tolerant control of an asynchronous machine", The Mediterranean Journal of measurement and control, Vol.10, No.3, pp.284-291, 2014.

[16] D.J. Khodja, S. Belhamdi, and B. Bendjaima, "Vector Control of Induction Machine with Models Taking Into Account the Stator and Rotor Faults", International Science and Technology Conference ISTEC'2011, Istanbul Turkey, pp.944-947, 2011. 